\title{
Spatial accessibility of primary health care utilising the two step floating catchment area method: an assessment of recent improvements
}

Matthew R McGrail ${ }^{1,2^{*}}$

\begin{abstract}
Background: The two step floating catchment area (2SFCA) method has emerged in the last decade as a key measure of spatial accessibility, particularly in its application to primary health care access. Many recent 'improvements' to the original 2SFCA method have been developed, which generally either account for distance-decay within a catchment or enable the usage of variable catchment sizes. This paper evaluates the effectiveness of various proposed methods within these two improvement groups. Moreover, its assessment focuses on how well these improvements operate within and between rural and metropolitan populations over large geographical regions.

Results: Demonstrating these improvements to the whole state of Victoria, Australia, this paper presents the first comparison between continuous and zonal (step) decay functions and specifically their effect within both rural and metropolitan populations. Especially in metropolitan populations, the application of either type of distance-decay function is shown to be problematic by itself. Its inclusion necessitates the addition of a variable catchment size function which can enable the 2SFCA method to dynamically define more appropriate catchments which align with actual health service supply and utilisation.
\end{abstract}

Conclusion: This study assesses recent 'improvements' to the 2SFCA when applied over large geographic regions of both large and small populations. Its findings demonstrate the necessary combination of both a distance-decay function and variable catchment size function in order for the 2SFCA to appropriately measure healthcare access across all geographical regions.

Keywords: Spatial accessibility, Primary health care, Rural health, Access to health care, Service catchments, Medical geography

\section{Introduction}

Access to health care is widely accepted internationally as a key goal in meeting the health needs of individuals [1-4]. However, assessing the extent to which adequate access to health care services is achieved is difficult because there is no single agreed definition of access [5-8]. Healthcare access is such a complex concept that Norris and Aiken [9] went as far as to state that "It is as if everyone is writing about 'it' [access] but no one is saying what 'it' is".

Correspondence: matthew.mcgrail@monash.edu

'Gippsland Medical School, Monash University, Northways Road, Churchill, VIC 3842, Australia

${ }^{2}$ Centre of Research Excellence in Rural and Remote Primary Health Care, Gippsland, Australia
A fundamental problem of defining access is its status as both a noun and a verb [10], thus healthcare access can refer both to the potential for use as well as the act of using healthcare. Furthermore, access is multidimensional with specific access barriers covering a range of spatial and aspatial dimensions [11-13], making it difficult to operationalise. Health service planners have tended to adopt Penchansky and Thomas' [13] five main dimensions of access - specifically availability, accessibility, affordability, accommodation and acceptability. As a result, healthcare access indicators vary immensely, and may be capturing but not limited to the availability of care, the ability to get to and pay for available care, or the act of seeking and utilising available care. One common approach to evaluating access to health care is
C Biomed Central

(c) 2012 McGrail; licensee BioMed Central Ltd. This is an Open Access article distributed under the terms of the Creative Commons Attribution License (http://creativecommons.org/licenses/by/2.0), which permits unrestricted use, distribution, and reproduction in any medium, provided the original work is properly cited. 
through measuring spatial accessibility [10,14-16]. Spatial accessibility provides a summary measure of two important and related components of access - firstly the volume of services provided relative to the population's size and secondly the proximity of services provided relative to the location of the population. This paper focuses on one such measure of spatial accessibility, the two-step floating catchment area (2SFCA) method and evaluates its many recent 'improvements'.

\section{Background}

Spatial accessibility and the two-step floating catchment area (2SFCA) method

The accurate measurement of spatial accessibility to health care is problematic chiefly because there is seldom any predetermined assignment or single pathway between individuals and specific health care services. That is, in most western societies individuals are free to access health care wherever and from whomever they choose. Thus an assessment of available services relative to the needs of the population specific to a local area is challenging. This is especially true for primary health care services, the key facilitator of access within most international health care systems $[17,18]$, which co-exist in a network of overlapping catchments 'competing' for the population's utilisation of their services.

The two-step floating catchment area (2SFCA) method, pioneered by Luo and Wang [11,15,19], emerged from a background in which the shortcomings of existing measures of spatial accessibility were readily apparent. In particular, fundamental weaknesses of provider- or physician-population ratios (PPRs) are wellrecognised $[10,14,15]$, which fail to include both crossborder movement between boundaries and distance decay within boundaries, but most significant is their restriction to using fixed geographical or administrative boundaries such as counties or postcodes. The 2SFCA method builds upon the framework of PPRs, but instead uses floating catchment areas which overlap, thereby enabling the modelling and measurement of 'real-life' healthcare access behaviour with unrestricted utilisation. The size of the catchment is determined by a choice of maximum travel time (or distance), where all services (or populations) within that catchment are considered accessible and equally proximate to that particular population (or service), whilst all locations outside of the catchment are not accessible.

The process for calculating the 2SFCA method is relatively straightforward. Step 1 of the 2SFCA method determines what populations $(\mathrm{k})$ of size $\mathrm{P}_{\mathrm{k}}$ are located within the catchment of each service provider (j) of volume $S_{j}$, thus defining the provider-to-population ratio $R_{j}$ within a service catchment (that is, the potential service demand). Step 2 then 'allocates' these service ratios to the population by determining which services (j) are located within the catchment of each population (i), and aggregating the Step $1\left(\mathrm{R}_{\mathrm{j}}\right)$ scores to calculate a location's access $\left(\mathrm{A}_{\mathrm{i}}\right)$. The only decision required in applying the 2 SFCA method is the catchment size $\left(\mathrm{d}_{\max }\right)$, which is then applied at both Steps 1 and 2 . This method has been utilised in this form or with minor modifications only, within the last six years by many different studies [20-31].

Step 1: For each service (j), $R_{j}=S_{j} / \sum_{k \in\{d j k<d m a x} P_{k}$

Step 2: For each population (i), $\left.A_{i}=\sum_{j \in\{d i j<d m a x}\right\}$

The following assumptions are made regarding application of the 2SFCA method:

- Service providers are represented by their geocoded organisational address (latitude, longitude).

Aggregating service counts to some administrative boundary (e.g. town, county, postcode) will simplify its computation, but can greatly reduce its sensitivity to small-area discrimination.

- Population (aggregated) groups are represented through a single location (centroid, usually geometric or population-weighted), based on some larger administrative boundary. Usage of smaller areal units enables more accurate small-area measurement of 'local' access, but also greatly increases computation complexity.

- Population-provider proximity (d) is measured as time or distance separation (point-to-point) through some transport network (roads, public transport). Euclidean distance can also be used to approximate proximity; however, this results in a moderate loss of accuracy.

Whilst the greatest strength of the 2SFCA method is it overcomes the restriction of using only pre-defined regional boundaries, this improvement alone does not address two major weaknesses still apparent in its framework. Firstly, distance-decay is assumed to be negligible within a catchment, something which is clearly not the case in large geographical regions where populations are widely dispersed, and catchments therefore are quite extensive. Secondly, catchments are assumed to be the same size for all populations and for all services.

Over the past five years, several authors have developed methodological 'improvements' to address these weaknesses characterising the 2SFCA method. This paper assesses these improvements to the 2SFCA method by evaluating their effectiveness when applied to primary health care access. Moreover, this assessment will focus on how well these improvements operate within and between rural, regional and metropolitan populations over large geographical regions. Health policies are mostly applied at national or state levels which require methodologies to work across diverse and large geographies. However, to date most 2SFCA method 
improvements have been demonstrated only within small or localised areas.

\section{Improvement 1 - addition of distance decay function}

Without the addition of a distance decay function, there is widespread agreement that the 2SFCA method is deficient [32-35]. Its omission is equivalent to accepting that distance (or time) is a negligible barrier within a catchment, an unlikely scenario for geographically large countries and given our use of a maximum catchment of 60 minutes in this paper. Within a service catchment (Step 1), distancedecay omission means that any service is equally likely to be delivering services to both populations very close by and those up to the catchment boundary (60 minutes). Within a population catchment (Step 2), distance-decay omission means that individuals are equally likely to be accessing services from both nearby and up to the catchment boundary (60 minutes). Whilst omission of both of these may be acceptable for a small scale model in a densely populated area (such as within major cities), it is clear that a distance-decay function is crucial in sparsely populated (rural) areas where problems associated with poor access to health care services are known to be a major factor contributing to the poorer health status of population in these areas [36,37].

Currently there is little empirical evidence to guide the choice of one decay function over another. Wang [38] defined six different distance-decay functions, where the crude 2SFCA method is defined by its use of a binary discrete function with no decay within a catchment and complete decay outside of a catchment. Luo \& Qi [34] developed what they called the 'enhanced' 2-step floating catchment area (E2SFCA) method, where catchments are broken into 3 discrete zones (0-10 minutes; $11-20$ minutes; 21-30 minutes) with constant weightings $(\mathrm{w}<=1)$ applied to the accessibility within each zone. Subsequently, some authors have accepted the E2SFCA method as the new 'standard' 2SFCA method [21,32,39], whilst Wan et. al. [40] extended this approach by adding a 30-60 minutes zone. Drawing on Wan and the Gaussian distribution [40,41], two sets of weightings are tested in this paper for these four time barrier zones, relating to either fast or slow decay:

- Fast step-decay: weightings $(\mathrm{w})=1,0.60,0.25,0.05$

- Slow step-decay: weightings $(\mathrm{w})=1,0.80,0.55,0.15$.

In applying this zonal or step approach to large geographical areas, the key criticism remains - specifically that accessibility weightings are equal within each zone and there is a sudden step (drop) at the edge of each zone [32,33], something which does not match real utilisation behaviour. Resultantly, many authors have developed distance-decay functions which are smoother and continuous in their decay $[32,35,42,43]$; however without any empirical evidence, it is unclear which function is the most appropriate to use [38]. This paper tests one such continuous weighting function:

- Continuous decay: weightings $(\mathrm{w})=1$ for the first 10 minutes, $\mathrm{w}=0$ for more than 60 minutes, and $\mathrm{w}$ $=((60-\mathrm{d}) /(60-10))^{\wedge} 1.5$ for distance/time $(\mathrm{d})$ between 10 and 60 minutes.

Previous testing suggested 1.5 was an appropriate weighting factor [35], though higher values such as 2 would achieve a quicker decay rate. Figure 1 shows the relative weightings of the three decay functions to be tested.

\section{Improvement $\mathbf{2}$ - addition of variable catchment size function / variable inclusion of distance decay}

One feature of applications of the 2SFCA method by different authors is their choice of different catchment sizes, with those in metropolitan settings generally using significantly smaller catchment sizes than for rural settings. Metropolitan and rural regions have very different settlement patterns, meaning that the appropriate catchment size for different regions is likely to vary greatly. Despite this, to date only two research groups have investigated the use of a variable catchment size function or the related variable inclusion of a distance decay function [35,44], with each using very different methods for defining their population and service catchment sizes.

Variable population catchment size (Step 2) is conceptually simple - individuals can travel further (or have a higher likelihood of travelling further) to access healthcare if nearby services do not meet their needs [45]. In metropolitan areas, services and populations are densely located so that, typically, most individuals will access services in close proximity because their requirements are met. In contrast, rural services are more dispersed, so that rural populations commonly access services beyond their immediate community. McGrail and Humphreys [46] modelled this $\mathrm{rural} /$ metropolitan distinction by limiting population catchment sizes to those containing the nearest 100 services (up to a maximum of 60 minutes, with a minimum catchment of 10 minutes). Similarly, Luo and Whippo [44] defined a minimum population catchment size of 10 minutes; however their approach was very different as a result of incrementally increasing the population catchment size (up to a maximum of 60 minutes) until a minimum provider-topopulation ratio $(1: 3500)$ is reached.

In metropolitan settings, both of these approaches ensure that the population catchment size is close to the minimum level of 10 minutes. In rural settings, McGrail's approach will have negligible effect because most rural populations will not have 100 services within a catchment 


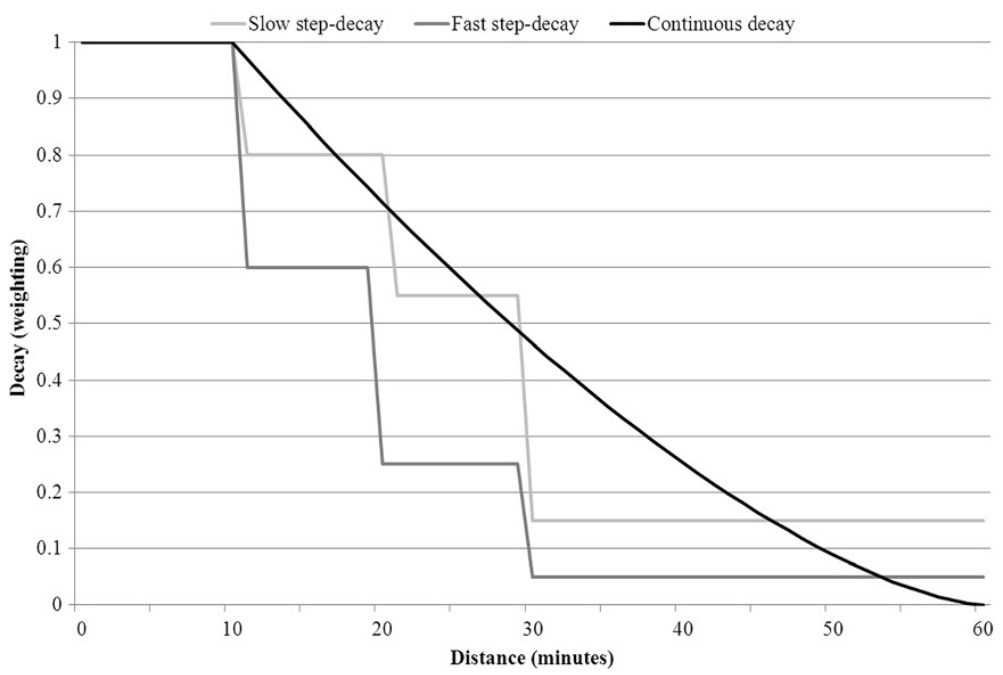

Figure 1 Decay function (weighting) versus distance (minutes).

of up to 60 minutes; however in metropolitan-fringe settings the effect of this approach will be most noticeable because the capping of 100 nearest services will vary greatly. Luo's approach does not distinguish between geographical settings; rather its effect is dependent on the local access level with populations modelled as only travelling further if their local access is below a minimum level.

Variable service catchment size (Step 1) is conceptually more difficult, with three broad scenarios defining different catchment areas requirements: (1) Metropolitan services provide access mostly to only their local neighbourhood; (2) Services in metropolitan-fringe areas or larger rural communities will frequently serve populations located well beyond the local community; (3) In contrast, services in small rural communities are generally not providing access for populations in larger nearby communities, who have adequate access within their immediate community.

McGrail and Humphreys proposed a method for variably applying distance decay at Step 1 across all regions, based on differentiating local population distributions [35]. Their four sequential rules (Table 1) are broadly designed to follow the three scenarios described in the previous paragraph and enable the 2SFCA method to dynamically determine where distance-decay should be applied (and thus reduce the size of the population being served).

Luo and Whippo [44] took a much simpler approach to defining service catchments by increasing its size incrementally (starting at 10 minutes) until the catchment population reaches 500,000 (note: for this paper, 250,000 or $250 \mathrm{~K}$ was considered a more appropriate size, and tested henceforth). It is clear that Luo's approach will have minimal or no effect on service catchments in sparsely populated (rural) areas, where the catchment population will be significantly less than $250 \mathrm{~K}$.

\section{Methods}

Study area and data requirements

To evaluate the 2SFCA method improvements, access scores are calculated using general practitioner (GP) service data in the state of Victoria, Australia (see Figure 2). Victoria has a total area of 227,000 square kilometres and 2011 population in excess of $5 \frac{1}{2}$ million (of which some 35\% reside outside of its major city of Melbourne). Firstly, population size and location data were obtained from the 2006 national census, using the smallest geographical unit of 'collection districts' (CDs) which contain an average population of approximately 500

Table 1 McGrail and Humphrey's rules to define the variable application of distance-decay to service catchments (Step 1)

\begin{tabular}{|c|c|c|}
\hline Rule & Explanation & Outcome \\
\hline 1. Population within 10 minutes of the service & Initial (local neighbourhood) catchment without distance decay & No decay \\
\hline 2. Population linked to their nearest 25 services & $\begin{array}{l}\text { Services likely to provide access to populations (beyond } 10 \text { minutes) } \\
\text { that have few alternative options }\end{array}$ & No decay \\
\hline $\begin{array}{l}\text { 3. Population }<5000 \text { and }<0.5 \text { population of } \\
\text { the Service town }\end{array}$ & $\begin{array}{l}\text { Services in larger towns likely to provide access to significantly } \\
\text { smaller nearby populations, but not vice-versa }\end{array}$ & No decay \\
\hline 4. All other scenarios & Services less likely to provide access to populations as distance increases. & Decay \\
\hline
\end{tabular}




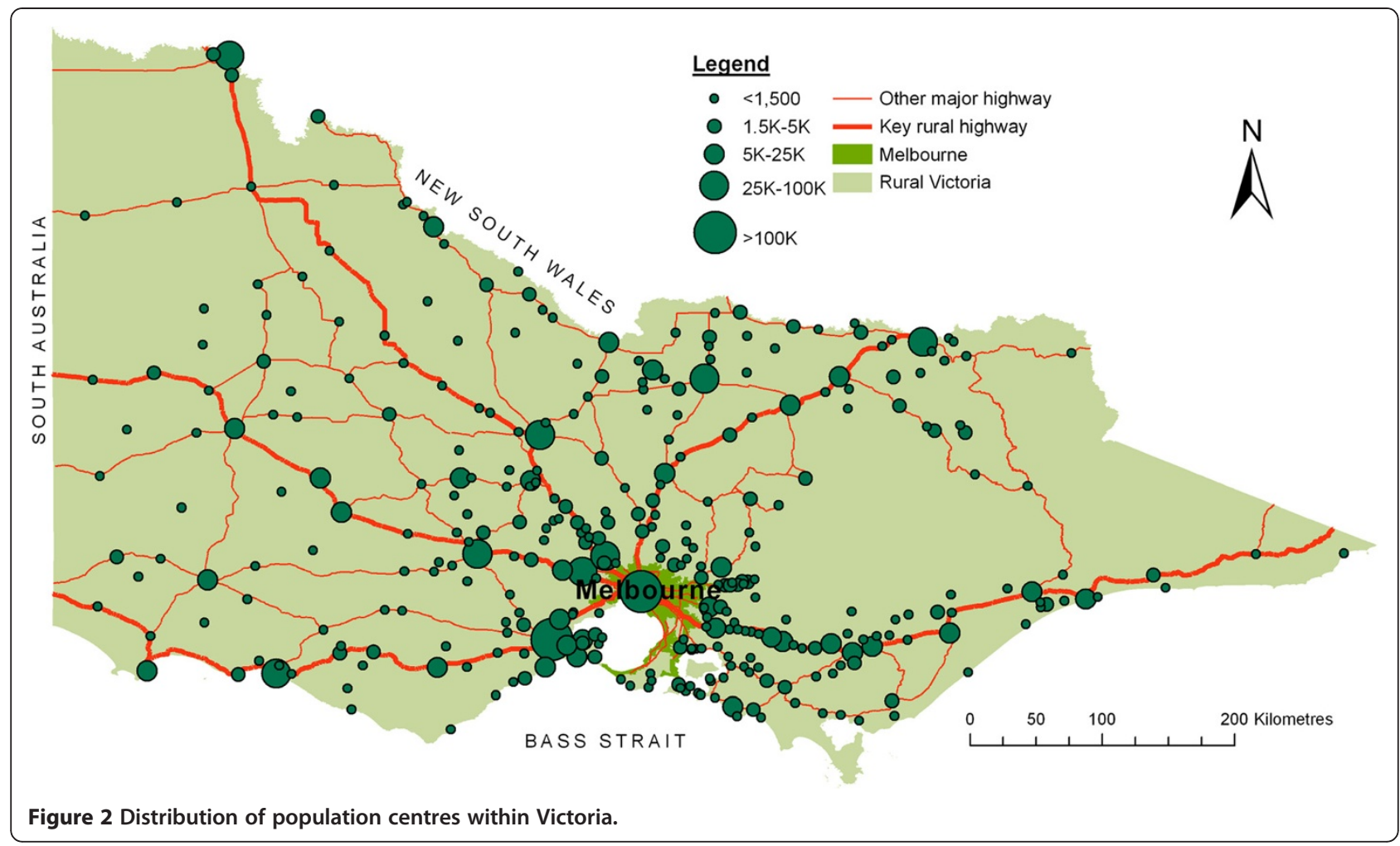

residents. Secondly, lists of GP locations (street address) and full-time equivalence counts were obtained from the Medical Directory of Australia (2006 records), a dataset which is updated every six months and promoted as being over $99 \%$ accurate and over $90 \%$ complete. Thirdly, proximity between geo-coded GP locations and population (CD) centroids was calculated using road networks and the 'Closest Facility' tool of the Network Analysis module of ArcView 9.1, with travel time impedance captured by combining road section lengths and approximate section travel speeds. Based on the notion of the 'golden hour' rule [47,48], a maximum catchment size of 60 minutes was used, and bordering data up to one catchment in width, from the neighbouring states of New South Wales and South Australia, were also included to take account of cross-border access at the edges of the study area.

Resultant access scores were calculated using the original (crude) 2SFCA method in combination with tested improvements as previously described. As a baseline comparison, Figure 3 shows the results of applying Luo and Wang's crude 2SFCA method across the whole state of Victoria, that is, before the inclusion of any improvements'. All access scores are assessed against a five-level community population size scale, which align closely with natural break points of primary care service provision in Australia [49,50]. Specifically, these are: (1) very small rural: $<1,500$ residents (none or limited GP services within community); (2) small rural: 1,500-4,999 residents (narrow choice of GP services within community); (3) medium rural: 5,000-24,999 residents (moderate choice of GP services within community); (4) large rural: 25,000-99,999 residents (wide choice of GP services within community); (5) metropolitan: $>100,000$ residents.

\section{Results}

Outcomes: improvement 1 - addition of distance decay function

Table 2 and Figure 4 show the result of integrating each of three different distance-decay functions within the 2SFCA method at both Step 1 and Step 2, which are summarised across five population size groupings. Within the four rural groupings $(<100 \mathrm{~K})$, there is a consistent pattern between decreasing population size and increasing proportion of lower access scores (see Table 2) following the addition of any distance-decay function. This is most apparent in the very small rural group $(<1.5 \mathrm{~K})$, where most populations do not have a local resident doctor and thus are most affected by the introduction of a distancedecay function. As a result, a large shift of access scores towards the lowest 4 categories $\left(7^{\text {th }}-10^{\text {th }}\right)$ was seen in $<1.5 \mathrm{~K}$ communities for all three distance-decay functions. In contrast, the large rural group $(25-100 \mathrm{~K})$ saw a shift of access scores towards the higher access categories $\left(2^{\text {nd }}-4^{\text {th }}\right)$ and a decrease in the poorer access $\left(5^{\text {th }}-7^{\text {th }}\right)$ categories. The addition of the distance decay 


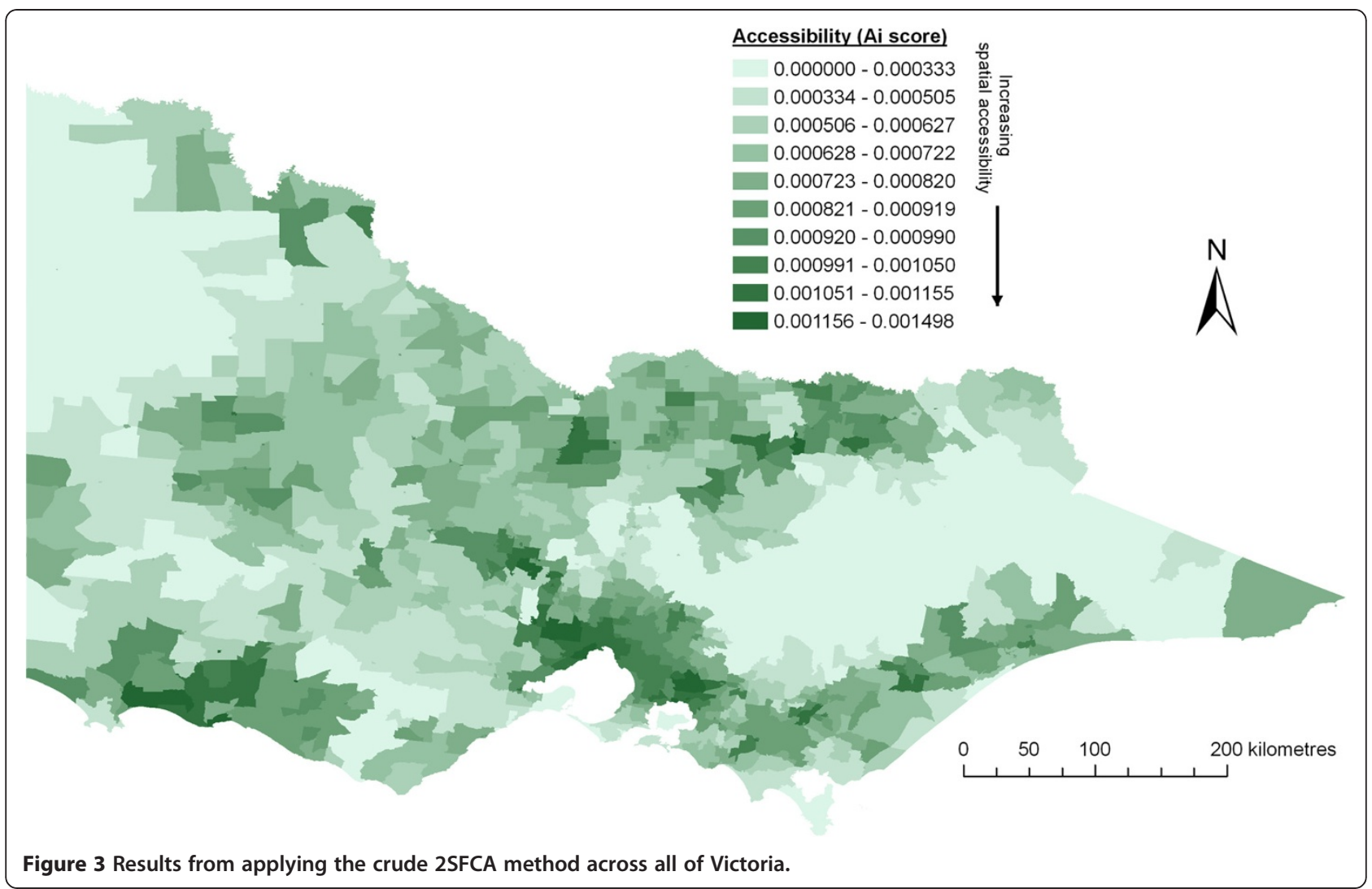

function has strengthened the association between both large populations and increased access, and between small populations and decreased access.

The three distance-decay functions revealed only minor differences to the change of access scores in Table 2 across the four rural groupings, though it must be noted these are net population changes and don't necessarily apply to the same geographic regions. In particular, there is a high correlation for changes between the slow step-decay and continuous decay functions. Figure 1 shows that these functions have similar shapes thus their level of similarity in resultant access scores is not altogether surprising. Closer assessment reveals a slight bias towards higher access scores in rural populations using the continuous function, which is likely due to its slower decay beyond 30 minutes. Specific to the fast step-decay function, two moderate differences are observed. Firstly, in the smallest rural group $(<1.5 \mathrm{~K})$ there is a considerable increase in the size of the poorest access group. This occurs because as distance-decay is applied more quickly (at Step 2), populations who are required to travel further to access distant services are affected most and thus their access scores decrease most. The second noticeable difference in rural populations is a small increase in the two highest access categories $\left(1^{\text {st }}\right.$ and $\left.2^{\text {nd }}\right)$. This occurs because a faster distance-decay (applied at Step 1) causes decreased demand from distant populations (that is, it decreases the denominator in Equation 1), the result of which is inflated access scores.

The introduction of a distance decay function has had the greatest effect (population size) within metropolitan centres $(>100 \mathrm{~K})$. This is largely explained by the crude 2SFCA method allocating most metropolitan populations to only the $2^{\text {nd }}$ and $3^{\text {rd }}$ access categories. After applying any one of the distance-decay functions, the highest access category has increased greatly $(694 \mathrm{~K}-1039 \mathrm{~K})$, mostly at the expense of the $2^{\text {nd }}$ access category whilst the $4^{\text {th }}$ and $5^{\text {th }}$ categories have increased significantly $(142 \mathrm{~K}-306 \mathrm{~K})$ at the expense of the $3^{\text {rd }}$ access category. Meanwhile, the lowest 5 access categories for metropolitan populations have all increased slightly, with the fast step-decay function seeing the largest increase in poorer access scores. Notably, Figure 4 demonstrates a very strong geographical pattern within metropolitan Melbourne, with the largest access gains located in the geographical centre whilst the largest losses are all located in the outer-urban / ruralfringe areas with a clear concentric pattern of decreasing 'access' from the centroid to the metropolitan-fringe. This suggests that with the addition of distance-decay function, the 2SFCA method is now largely measuring 'choice' in metropolitan areas. In the next section, which assesses the 
Table 2 Distribution of crude access (2SFCA) scores and resulting change of access scores with the addition of three different distance-decay functions, by population size

\begin{tabular}{|c|c|c|c|c|c|c|c|c|c|c|}
\hline & & & & & cess catego & $\left(A_{i}\right.$ score $r$ & ge) & & & \\
\hline & $1>0.0012$ & $2>0.001$ & $3>0.0009$ & $4>0.0008$ & $5>0.0007$ & $6>0.0006$ & $7>0.0005$ & $8>0.0004$ & $9>0.0003$ & $10<0.0003$ \\
\hline Crude acc & ss (2SFCA) & ore distrib & tion $\wedge 1$ & & & & & & & \\
\hline$>100 \mathrm{~K}$ & 17 & 2012 & 1049 & 17 & 64 & 59 & 10 & 23 & 0 & 10 \\
\hline $25-100 K$ & 28 & 0 & 34 & 61 & 38 & 99 & 49 & 0 & 0 & 0 \\
\hline $5-25 K$ & 12 & 44 & 66 & 55 & 30 & 27 & 23 & 26 & 3 & 18 \\
\hline $1.5-5 \mathrm{~K}$ & 0 & 32 & 28 & 34 & 24 & 15 & 19 & 7 & 3 & 11 \\
\hline$<1.5 \mathrm{~K}$ & 10 & 44 & 67 & 92 & 66 & 85 & 61 & 44 & 34 & 30 \\
\hline Slow step & decay (net & ange from & rude $2 \mathrm{SFCA}$ & $\wedge 2$ & & & & & & \\
\hline$>100 \mathrm{~K}$ & 992 & -1115 & -484 & 182 & 302 & 4 & 42 & 46 & 29 & 1 \\
\hline $25-100 K$ & -28 & 63 & 45 & 21 & -32 & -45 & -49 & 25 & 0 & 0 \\
\hline $5-25 \mathrm{~K}$ & -2 & 4 & -16 & -10 & -12 & 19 & 44 & -8 & -2 & -18 \\
\hline $1.5-5 \mathrm{~K}$ & 6 & -17 & -11 & -11 & 3 & 7 & -6 & 15 & 20 & -5 \\
\hline$<1.5 \mathrm{~K}$ & -7 & -26 & -36 & -45 & -2 & -21 & 27 & 38 & 28 & 44 \\
\hline
\end{tabular}

Continuous decay (net change from crude access scores) $\wedge 2$

\begin{tabular}{|c|c|c|c|c|c|c|c|c|c|c|}
\hline$>100 \mathrm{~K}$ & 694 & -878 & -374 & 306 & 190 & -23 & 11 & 36 & 37 & 1 \\
\hline 25-100K & -28 & 51 & 45 & 33 & -32 & -45 & -49 & 25 & 0 & 0 \\
\hline $5-25 K$ & -3 & 17 & -19 & 2 & 7 & 28 & 7 & -25 & 5 & -18 \\
\hline $1.5-5 \mathrm{~K}$ & 4 & -18 & -11 & -2 & 3 & 8 & -2 & 18 & 1 & -2 \\
\hline$<1.5 \mathrm{~K}$ & -6 & -21 & -38 & -35 & 12 & 3 & 6 & 30 & 13 & 37 \\
\hline
\end{tabular}

Fast step-decay (net change from crude access scores) $\wedge 2$

\begin{tabular}{|c|c|c|c|c|c|c|c|c|c|c|}
\hline$>100 \mathrm{~K}$ & 1039 & -1338 & -344 & 165 & 142 & 188 & 84 & 49 & 14 & 1 \\
\hline $25-100 K$ & -25 & 113 & 54 & -44 & -2 & -99 & -21 & 0 & 25 & 0 \\
\hline $5-25 K$ & 3 & 32 & -19 & -17 & -20 & 46 & -3 & -20 & 15 & -17 \\
\hline $1.5-5 \mathrm{~K}$ & 10 & -12 & -9 & -12 & -2 & -2 & -9 & 22 & 10 & 6 \\
\hline$<1.5 \mathrm{~K}$ & -1 & -23 & -45 & -59 & -16 & -25 & -10 & 44 & 39 & 95 \\
\hline
\end{tabular}

All figures within the table are '000s.

1: These values represent the size ('000s) of the population with these access scores. Row totals correspond to the total Victorian population residing in the 5 population size groups.

^2: These values represent the net population change ('000s) within each population size group to the corresponding access scores following the addition of each distance-decay function. Negative values indicate a net drop in the number of residents with access scores in that category. All row totals equal 0.

addition of a variable catchment size function, the slow step-decay function has been included.

\section{Outcomes: improvement 2 - addition of variable} catchment size function / variable inclusion of distance decay

Figures 5 and 6 respectively show the change to access scores across different geographic regions with the addition of either Luo's or McGrail's variable catchment size function. Outcomes from applying these functions are further summarised across five population size groupings in Table 3 and many differences between these functions are apparent.

Arguably the greatest need for a variable catchment size function is within metropolitan-fringe areas. It was seen in Figure 4 that the resultant change from applying a distance-decay function alone in these areas was consistently decreasing access scores moving out from the city centre to metropolitan-fringe populations. This is chiefly because the 2SFCA method, prior to adding a variable catchment size function, models metropolitan-fringe locations as being 'swamped' by inner-metropolitan populations travelling out to these fringe (and nearby rural) locations, guaranteeing their lower access scores. Both functions (Luo's and McGrail's) rectify this problem to varying degrees. McGrail's Step 1 distance-decay function rules (Table 1) have reversed much of the drop in access scores in metropolitan-fringe areas, though its validity is difficult to assess. Luo's simpler approach too increases access in many metropolitan-fringe areas, but is less effective for two reasons. Firstly, their constant use of a $250 \mathrm{~K}$ service catchment ensures metropolitan-fringe populations 


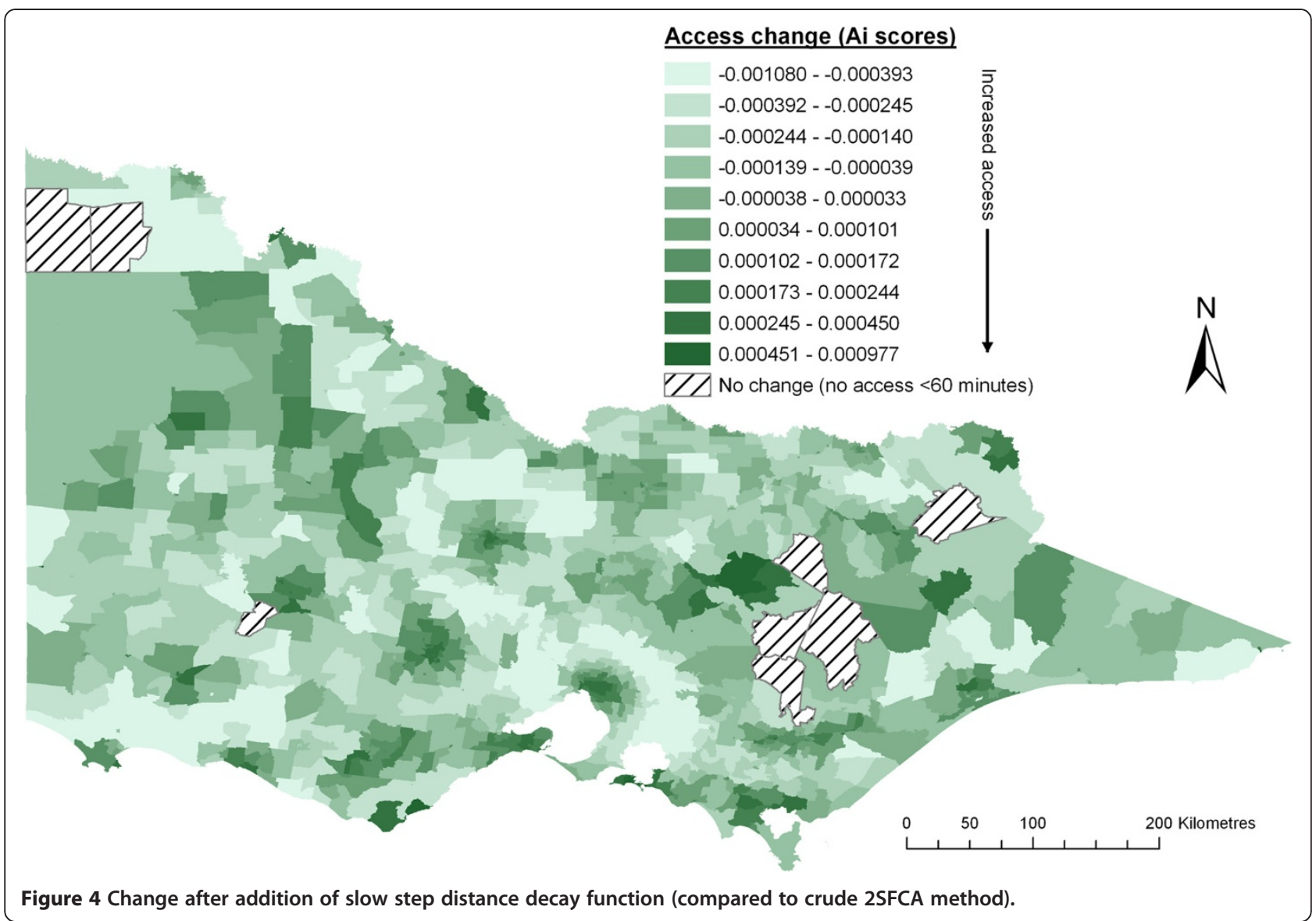

continue to 'swamp' nearby rural services. Secondly, their function which expands the population catchment only until a minimum access level $(<1: 3500)$ is reached is problematic because in nearby rural areas it is highly likely that local populations can and will choose to travel a little further into metropolitan-fringe areas to access services. Thus the 'real' access score should be larger in these areas than their function allows.

In all rural population groups, Table 3 shows that Luo's approach has reduced access levels for more of the population compared to McGrail's approach. For example, in 5 -25K rural towns Luo's model has increased a net total of $126 \mathrm{~K}$ into the lowest 6 access categories $(<0.0008)$ whilst the comparative net movement in McGrail's model to the same 6 access categories is 59K. This pattern is consistent in all five population size groupings, to various degrees and is predominantly due to Luo's use of the population catchment size minimum PPR rule ( $>1: 3500$ as applied to Step 2), whilst their Step 1 rule has virtually no effect in rural areas. Interestingly, the opposite application occurs in McGrail's model with their rule of limiting the population catchment to the nearest 100 services (as applied to Step 2) having virtually no effect in rural areas, whilst their Step 1 rules (Table 1) greatly affect rural access scores.
Visually, changes in rural access scores are quite different using either Luo's (Figure 5) or McGrail's (Figure 6) approach. Closer investigation of Figure 6 (McGrail's approach) reveals that many areas showing the largest decrease in access accord directly with areas of largest increase in access in Figure 4. That is, for many areas there is a direct 'correction' from first applying the distance-decay function, then adding the variable catchment size function. This is explained by the Step 1 rules (Table 1) which purposefully aim to improve the determination of when, or if, the distance-decay function should be applied. Resultant access changes from Luo's approach (Figure 5) have no apparent relationship to the distance-decay function (Figure 4).

\section{Discussion}

This paper has critically evaluated current and recent work by a number of different researchers who have developed new methodologies aimed at improving the 2SFCA method. Generally, these improvements aim to address one of two deficiencies of Wang and Luo's original (crude) 2SFCA method: (1) accounting for distance decay within a catchment; and (2) enabling variable catchment sizes or variable application of distance-decay. 


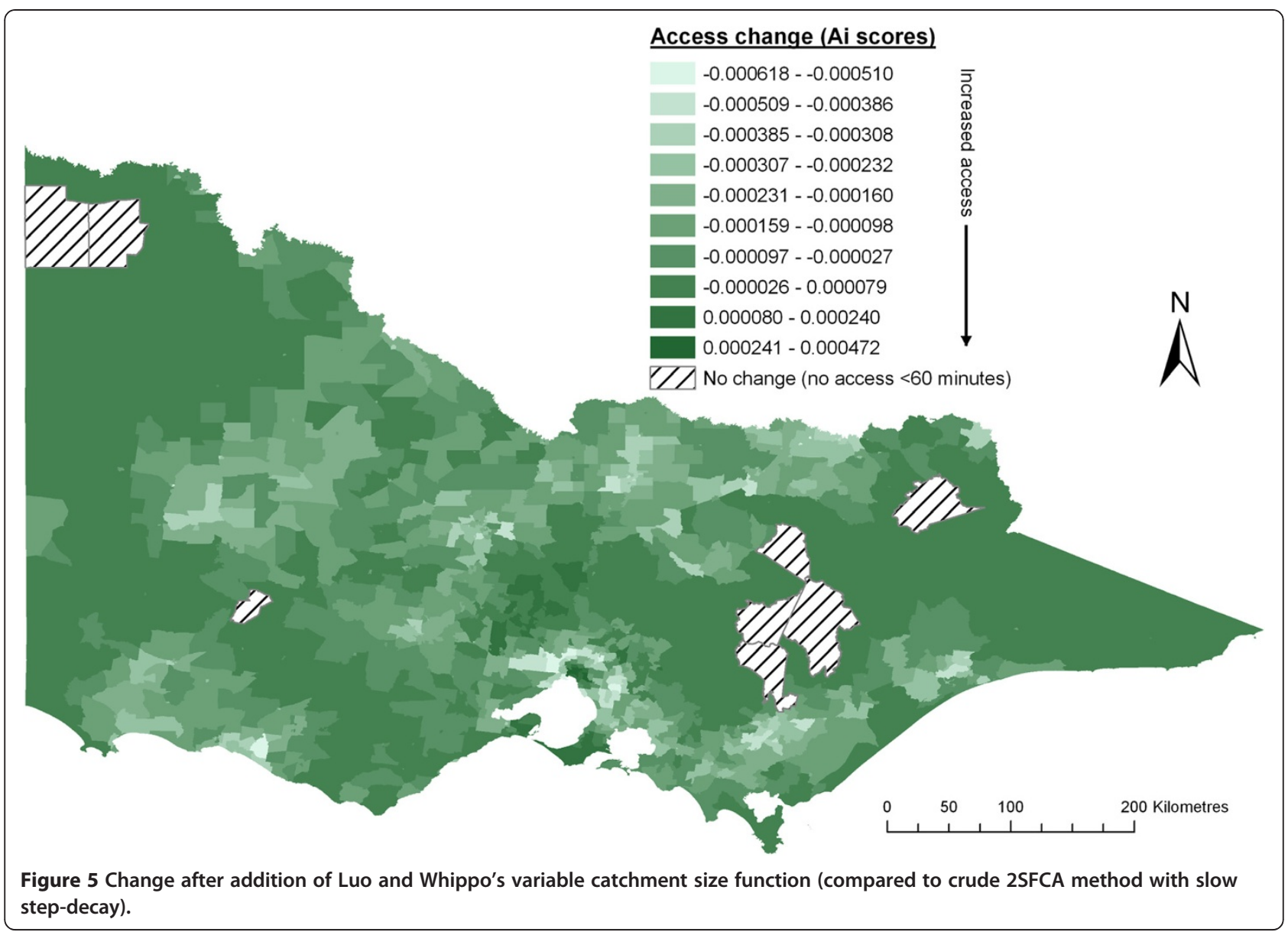

To date, most 2SFCA method improvements relate to the addition of a distance-decay function, with a general acceptance that its omission is highly problematic. This paper provides the first evidence of the effect of applying one of three related distance-decay functions, and most significantly their application across large geographic regions of both rural and metropolitan populations. Despite some criticisms of the step-decay function having a sudden drop in access at the edge of each zone, these results showed relatively minor differences when comparing the continuous and slow-zone functions, particularly in more sensitive rural areas. A continuous-decay function may intuitively be preferable to a step-decay function, but it is difficult to define an appropriately shaped function that matches 'real' behaviour of the population (chiefly because of poor empirical evidence for health care seeking behaviour). Figure 1 suggests that the decay weighting of the tested continuous function may be too slow, particularly for a distance barrier of 30-60 minutes. In contrast, the consequence of choosing a faster step-decay function in rural areas has (perhaps wrongly) increased the proportion of low-access scores within small rural areas $(<25 \mathrm{~K})$ and somewhat increased the proportion of high access scores in larger rural areas (25-100K).

Importantly, the application of any distance-decay function without the simultaneous use of a variable catchment size function creates a strong concentric pattern of high to low access scores out through metropolitan-fringe and into nearby rural areas. This 'overcorrection' in metropolitan-fringe areas can be rectified by adding a variable catchment size function, so that more realistic movements of these populations are captured in the modelling. Furthermore, it is not clear that distance / time barriers are viewed consistently by all populations within different geographical settings, thus it is questionable whether the same distance-decay function would apply to all. For example, residents in isolated and vastly settled areas are likely to have a higher 'tolerance' of travelling further to access services versus residents in closely settled or largely populated areas.

The respective variable catchment size functions used by either McGrail and Humphreys or Luo and Whippo approach the problem from contrasting sides of access calculation. McGrail's method limits population catchments (applied at Step 2) to the nearest 100 services (or 


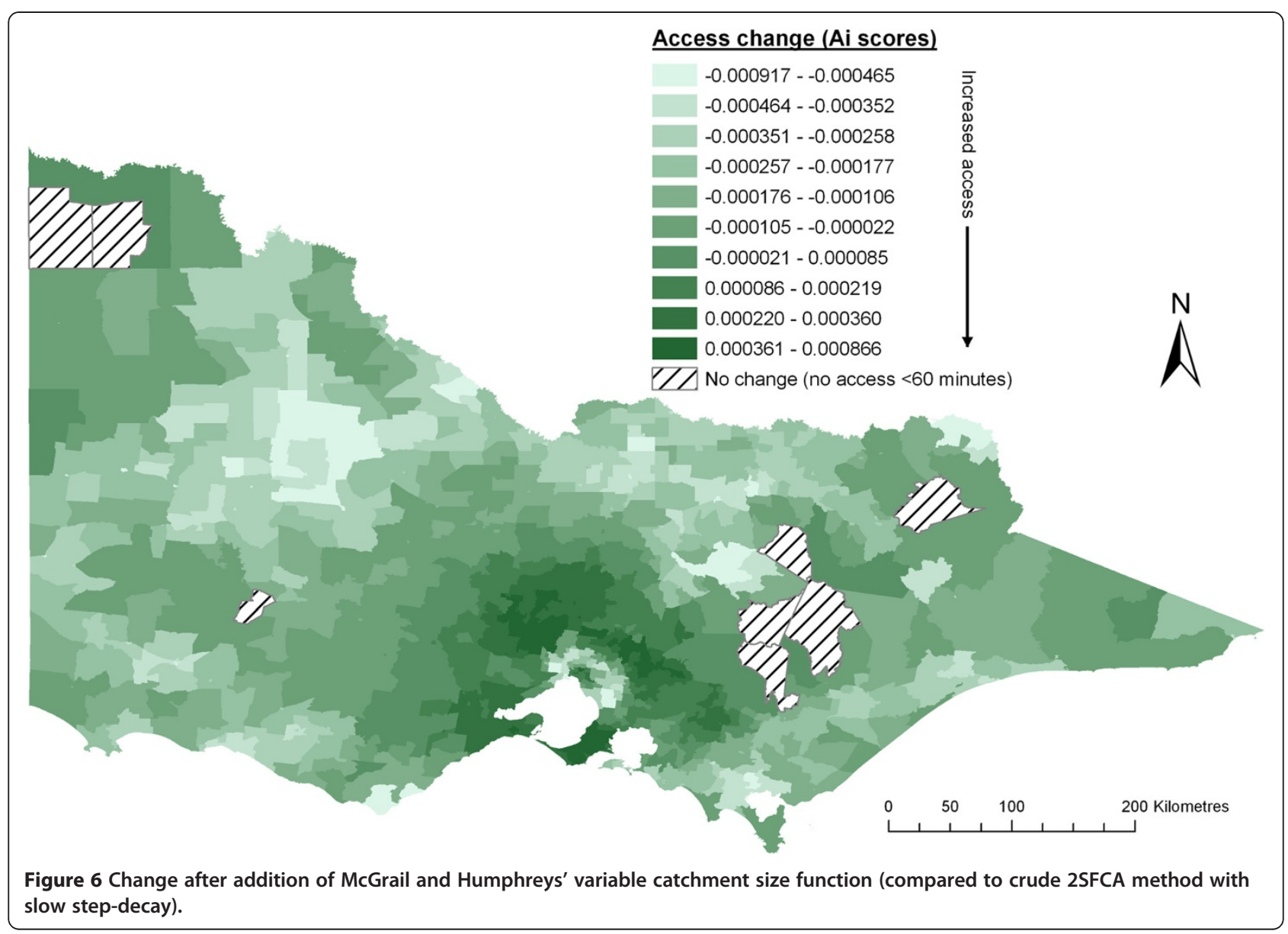

Table 3 Resultant change of access scores with the addition of two different variable catchment size functions, by population size

\begin{tabular}{|c|c|c|c|c|c|c|c|c|c|c|}
\hline & \multicolumn{10}{|c|}{ Access category ( $A_{i}$ score range) } \\
\hline & $1>0.0012$ & $2>0.001$ & $3>0.0009$ & $4>0.0008$ & $5>0.0007$ & $6>0.0006$ & $7>0.0005$ & $8>0.0004$ & $9>0.0003$ & $10<0.0003$ \\
\hline \multicolumn{11}{|c|}{ Luo and Whippo's approach (net change from crude access scores with slow-step decay)^1 } \\
\hline$>100 K$ & -250 & -600 & -521 & 127 & -74 & 255 & 624 & 326 & 125 & -11 \\
\hline $25-100 K$ & 0 & -63 & -79 & -55 & 67 & 67 & 34 & 29 & 0 & 0 \\
\hline $5-25 K$ & -10 & -47 & -44 & -25 & 21 & 46 & 13 & 17 & 28 & 1 \\
\hline $1.5-5 \mathrm{~K}$ & -6 & -9 & -6 & -11 & -13 & 5 & 32 & 0 & 1 & 7 \\
\hline$<1.5 \mathrm{~K}$ & -3 & -15 & -29 & -30 & -38 & 1 & 18 & 34 & 32 & 29 \\
\hline
\end{tabular}

McGrail and Humphreys' approach (net change from crude access scores with slow-step decay)^1

\begin{tabular}{|c|c|c|c|c|c|c|c|c|c|c|}
\hline$>100 K$ & -406 & -480 & -313 & 201 & 363 & 452 & 185 & 37 & -29 & -11 \\
\hline $25-100 K$ & 0 & -35 & -47 & 39 & 31 & 8 & 29 & -25 & 0 & 0 \\
\hline $5-25 K$ & -10 & -41 & -18 & 9 & 41 & 6 & -16 & 14 & 10 & 4 \\
\hline $1.5-5 \mathrm{~K}$ & -6 & -7 & -15 & 7 & -6 & 10 & 11 & 13 & -8 & 0 \\
\hline$<1.5 \mathrm{~K}$ & -2 & -11 & -17 & -14 & 7 & 4 & -13 & 2 & 13 & 30 \\
\hline
\end{tabular}

All figures within the table are '000s.

11: The base (comparison) model is a crude 2SFCA method with the addition of a slow step-decay function. These values represent the net population change ('000s) within each population size group to the corresponding access scores following the addition of each distance-decay function. Negative values indicate a net drop in the number of residents with access scores in that category. All row totals equal 0. 
minimum 10 minutes), whilst Luo's method limits service catchments (applied at Step 1) to the nearest $250 \mathrm{~K}$ residents (or minimum 10 minutes). Both of these approaches have full effect in metropolitan areas, no effect in rural areas and varying effect in metropolitanfringe and nearby rural areas. Luo's use of a minimum accessibility rule to define population catchment sizes (applied at Step 2) is problematic in how effectively it adjusts access scores. This was most apparent in metropolitan-fringe areas, where access scores were significantly lower using Luo's method, but it is arguable whether these populations would readily travel a small distance further to access metropolitan-based services areas, and so their access scores have been capped inappropriately. McGrail's approach to service catchments (applied at Step 1) does not use a single size for all populations, unlike Luo's constant use of $250 \mathrm{~K}$, but instead defines a set of four rules which account for neighbouring populations and service competition. Whilst these rules attempt to better match 'real' supply behaviour, further independent assessment is required.

The key limitation to this study is the lack of available empirical data on 'real' health service access behaviour and its relationship to geography. The 2SFCA method requires assumptions of catchment size, distance-decay and the variable application of these across metropolitan and rural populations; however, to date most applications of the 2SFCA method have not been verified against empirical access behaviour data. An additional related limitation is that it's not known whether this paper's findings (based on the state of Victoria, Australia) readily translate to other geographical settings and countries.

Most academic papers describing methodological developments or improvements such as those for the 2SFCA method underestimate the importance of the specifics of the geography under consideration. One of the difficulties of calculating spatial accessibility is modelling across vastly different population densities and dispersions. A key strength of the 2SFCA method is that it can be readily applied to both metropolitan and rural populations. However, this 'flexibility' can also be one of its weaknesses when applied simultaneously across all geographies. Catchment size or distance-decay rules that readily apply in densely populated areas generally don't apply in sparsely populated areas, and vice-versa. For this reason, the inclusion of a variable catchment size methodology, which is missing from the new standard E2SFCA method, is vital to the wider application of the 2SFCA method to health policies relating to healthcare access and primary health care service provision. This paper has provided further evidence relating to the inclusion and choice of both a distance-decay function as well as a variable catchment size function, highlighting in particular that large scale applications of the 2SFCA method require closer attention to their defining of population and service catchments.

\section{Competing interests}

There are no competing interests.

\section{Acknowledgements}

The research reported in this paper is a project of the Centre of Research Excellence (CRE) in accessible and equitable primary health service provision in rural and remote Australia funded by the Australian Primary Health Care Research institute, which is supported by a grant from the Commonwealth of Australia as represented by the Department of Health and Ageing. The information and opinions contained in it do not necessarily reflect the views or policy of the Australian Primary Health Care Research Institute or the Commonwealth of Australia (or the Department of Health and Ageing). I would also like to acknowledge the invaluable support from Emeritus Professor John Humphreys, Monash University School of Rural Health.

Received: 18 September 2012 Accepted: 6 November 2012

Published: 16 November 2012

\section{References}

1. United Nations General Assembly: The universal declaration of human rights. [http://www.un.org/en/documents/udhr/index/html].

2. Grad FP: The preamble of the constitution of the World Health Organization. B World Health Organ 2002, 80:981-984.

3. Department of Health and Aged Care: Reforming the Australian health care system: the role of government. Canberra: DHAC; 1999.

4. President's Commission for the study of ethical problems in medicine and biomedical and behavioral research: Securing access to health care: The ethical implications of differences in the availability of health services. Washington DC: President's Commission; 1983.

5. Aday LA, Andersen RM: Equity of access to medical care: a conceptual and empirical overview. Med Care 1981, 19:4-27.

6. Rogers A, Flowers J, Pencheon D: Improving access needs a whole systems approach. Br Med J 1999, 319:866-867.

7. Ansari Z: A review of literature on access to primary health care. Aust J Prim Health 2007, 13:80-95.

8. Gulzar L: Access to health care. J Nurs Scholarship 1999, 31:13-19.

9. Norris TL, Aiken M: Personal access to health care: a concept analysis. Public Health Nurs 2006, 23:59-66.

10. Guagliardo MF: Spatial accessibility of primary care: concepts, methods and challenges. Int J Health Geogr 2004, 3:3.

11. Wang F, Luo W: Assessing spatial and nonspatial factors for healthcare access: towards an integrated approach to defining health professional shortage areas. Health Place 2005, 11:131-146.

12. Khan AA, Bhardwaj SM: Access to health care: a conceptual framework and its relevance to health care planning. Eval Health Prof 1994, 17:60-76.

13. Penchansky $R$, Thomas JW: The concept of access: definition and relationship to consumer satisfaction. Med Care 1981, 19:127-140.

14. McGrail MR, Humphreys JS: Measuring spatial accessibility to primary care in rural areas: improving the effectiveness of the two-step floating catchment area method. Appl Geogr 2009, 29:533-541.

15. Luo W, Wang F: Measures of spatial accessibility to health care in a GIS environment: synthesis and a case study in the Chicago region. Environ Plann B 2003, 30:865-884.

16. Langford M, Higgs $G$ : Measuring potential access to primary healthcare services: the influence of alternative spatial representations of population. Prof Geogr 2006, 58:294-306.

17. World Health Organisation: Declaration of Alma-Ata, International conference on primary health care; Alma-Ata, USSR. Geneva: WHO; 1978

18. Starfield B, Shi L, Macinko J: Contribution of primary care to health systems and health. Milbank Q 2005, 83:457-502.

19. Luo W: Using a GIS-based floating catchment method to assess areas with shortage of physicians. Health Place 2004, 10:1-11.

20. Bagheri N, Benwell GL, Holt A: Primary health care accessibility for rural Otago: "a spatial analysis". Online: Health Care Inform Rev; 2006.

21. Brown SR: Are Oklahoma city residents ok? A socio-spatial analysis of physicians and supermarkets via accessibility and affordability PhD. Department of Geography: Oklahoma State University; 2011. 
22. Langford M, Higgs G, Radcliffe J, White S: Urban population distribution models and service accessibility estimation. Comput Environ Urban 2008, 32:66-80.

23. McLafferty S, Wang F: Rural reversal? Rural-urban disparities in late-stage cancer risk in Illinois. Cancer 2009, 115:2755-2764

24. Ngui AN, Apparicio P: Optimizing the two-step floating catchment area method for measuring spatial accessibility to medical clinics in Montreal. BMC Health Serv Res 2011, 11:166.

25. Mao K, Li M, Huang Q, Chen C, Hu W, Chen D: Evaluation of spatial accessibility to town and rural in a GIS environment: a case study of Dachang county, 19th International Conference on Geoinformatics. Shanghai, China: Institute of Electrical and Electronics Engineers (IEEE); 2011.

26. Roeger LS, Reed RL, Smith BP: Equity of access in the spatial distribution of GPs within an Australian metropolitan city. Aust J Prim Health 2010, 16:284-290.

27. Yang D-H, Goerge R, Mullner R: Comparing GIS-based methods of measuring spatial accessibility to health services. J Med Syst 2006, 30:23-32.

28. Cervigni F, Suzuki Y, Ishii T, Hata A: Spatial accessibility to pediatric services. J Commun Health 2008, 33:444-448.

29. Liu Y, Wong SY, Jin T: Equality of spatial access to primary health services for Singapore's baby boomers. Asian Popul Stud 2009, 5:171-188.

30. Bell S, Wilson K, Bissonnette L, Shah Tl: Access to primary health care: Does neighborhood of residence matter? Ann Assoc Am Geog 2012, In Press.

31. Bissonnette L, Wilson K, Bell S, Shah TI: Neighbourhoods and potential access to health care: The role of spatial and aspatial factors. Health Place 2012, 18:841-853.

32. Dai D, Wang F: Geographic disparities in accessibility to food stores in southwest Mississippi. Environ Plann B 2011, 38:659-677.

33. Langford M, Fry $R$, Higgs $G$ : Measuring transit system accessibility using a modified two-step floating catchment technique. Int J Geogr Inf Sci 2012, 26:193-214

34. Luo W, Qi Y: An enhanced two-step floating catchment area (E2SFCA) method for measuring spatial accessibility to primary care physicians. Health Place 2009, 15:1100-1107.

35. McGrail MR, Humphreys JS: The index of rural access: an innovative integrated approach for measuring primary care access. BMC Health Serv Res 2009, 9:124.

36. Macinko J, Starfield B, Shi L: Quantifying the health benefits of primary care physician supply in the United States. Int J Health Serv 2007, 37:111-126.

37. Gorey KM, Luginaah IN, Holowaty EJ, Fung KY, Hamm C: Associations of physician supplies with breast cancer stage at diagnosis and survival in Ontario, 1988 to 2006. Cancer 2009, 115:3563-3570.

38. Wang F: Measurement, optimization and impact of health care accessibility: a methodological review. Ann Assoc Am Geog 2012 102:1104-1112

39. Zhan Q, Wang X, Sliuzas R: A GIS-based method to assess the shortage areas of community health service - Case study in Wuhan, China, 201 International Conference on Remote Sensing, Environment and Transportation Engineering (RSETE). Nanjing, China: Institute of Electrical and Electronics Engineers (IEEE); 2011

40. Wan N, Zhan FB, Zou B, Chow E: A relative spatial access assessment approach for analyzing potential spatial access to colorectal cancer services in Texas. Appl Geogr 2012, 32:291-299.

41. Wan N, Zou B, Sternberg T: A 3-step floating catchment area method for analyzing spatial access to health services. Int J Geogr Inf Sci 2012, 26:1073-1089.

42. Schuurman N, Berube M, Crooks VA: Measuring potential spatial access to primary health care physicians using a modified gravity model. Can Geogr 2010, 54:29-45.

43. Dai D: Black residential segregation, disparities in spatial access to health care facilities, and late-stage breast cancer diagnosis in metropolitan Detroit. Health Place 2010, 16:1038-1052.

44. Luo W, Whippo TL: Variable catchment sizes for the two-step floating catchment area (2SFCA) method. Health Place 2012, 18:789-795.

45. Humphreys J, Mathews-Cowey S, Weinand HC: Factors in accessibility of general practitioners in rural Australia. Med J Aust 1997, 166:577-580.

46. McGrail MR, Humphreys JS: A new index of access to primary care services in rural areas. Aust NZ J Pub Health 2009, 33:418-423.

47. Billi JE, Pai C-W, Spahlinger DA: The effect of distance to primary care physician on health care utilization and disease burden. Health Care Manage R 2007, 32:22-29.
48. Lerner EB, Moscati RM: The golden hour: scientific fact or medical "urban legend"? Acad Emerg Med 2001, 8:758-760.

49. Humphreys JS, MCGrail MR, Joyce CM, Scott A, Kalb G: Who should receive recruitment and retention incentives? Improved targeting of rural doctors using medical workforce data. Aust J Rural Health 2012, 20:3-10.

50. McGrail MR, Humphreys JS: Geographical classifications to guide rural health policy in Australia. Aust NZ Health Policy 2009, 6:28.

\section{doi:10.1186/1476-072X-11-50}

Cite this article as: McGrail: Spatial accessibility of primary health care utilising the two step floating catchment area method: an assessment of recent improvements. International Journal of Health Geographics 2012 $11: 50$

\section{Submit your next manuscript to BioMed Central and take full advantage of:}

- Convenient online submission

- Thorough peer review

- No space constraints or color figure charges

- Immediate publication on acceptance

- Inclusion in PubMed, CAS, Scopus and Google Scholar

- Research which is freely available for redistribution

Submit your manuscript at www.biomedcentral.com/submit
C Biomed Central 